\title{
Evaluation of Third Generation Anti-HCV Test Kit (SYNPEPTM HCV-EIA II) Using Sera of Inhabitants from HCV Hyperendemic Area
}

\author{
MIKI SHIRACHI, MICHIO SATA, HIROSHI SUZUKI, KUNITAKA FUKUIZUMI, \\ KYUICHI TANIKAWA, YUJI ITOH*, TAKAAKI MIZUMOTO*, SHIGENOBU KONDOH*, \\ MICHIKO KASAHARA** AND SACHIKO MANABE**
}

The Second Department of Medicine and *Clinical Laboratory Department, Kurume University School of Medicine, Kurume 830-0011, and

**Research and Development Department (Takahagi), Kyokuto Pharmaceutical Inc., Tokyo, Japan

\begin{abstract}
Summary: We examined the characteristics and usefulness of a third generation anti-HCV test kit, SYNPEPTM HCV-EIA II (Kyokuto Pharmaceutical Inc., Tokyo, Japan). The sera of inhabitants from a hepatitis $\mathrm{C}$ virus (HCV) hyperendemic area were used. The kit had even or more anti-HCV detection sensitivity and reproducibility than ORTHO HCV III ELISA Test System ${ }^{\mathrm{TM}}$ (Ortho-Clinical Diagnostic K.K., Tokyo, Japan) or HCV PHA 2nd Generation ${ }^{\mathrm{TM}}$ (Dinabot Co., Ltd., Tokyo, Japan). SYNPEPTM HCV-EIA II needed less total reaction time than other EIA kits, resulting in a simple procedure. Also, HCV RNA was detected in $90 \%$ of subjects who had a 7.5 or greater cut-off index (COI) of SYNPEPTM HCV-EIA II kit. In conclusion, SYNPEPTM HCV-EIA II require cheap cost and simple procedure and it could be applied to mass screening to find out HCV RNA positive persons who may need clinical care.
\end{abstract}

Key words hepatitis C virus (HCV), anti-HCV antibody, hyperendemic area of HCV

\section{INTRODUCTION}

Hepatitis $\mathrm{C}$ virus (HCV) infection frequently results in chronic hepatitis and in some progresses to cirrhosis as well as liver cancer [1]. Chronic HCV infection is related to $70-80 \%$ of liver cancers [2], thus hepatitis $\mathrm{C}$ diagnosis and treatment are recognized as important clinical issues [3].

Chiron Inc. initially cloned the HCV sequence in 1989 and developed an antibody assay system to confirm HCV infection using recombinant antigen (C100-3) of the non-structural region of HCV [4]. However, this assay system had limited sensitivity and specificity $[5,6]$. A second generation antibody assay system was developed by combining antigens of the HCV core and non-structural regions. Since February 1992 blood donors in Japan have been screened for anti-HCV by second generation antibody assay system and post-transfusion hepatitis rarely occurred thereafter [7]. Further effort to improve the assay system have yielded third generation anti-HCV test kits. Here we evaluated the characteristics and usefulness of SYNPEP ${ }^{\mathrm{TM}} \mathrm{HCV}$ EIA II using sera of inhabitants from HCV hyperendemic area.

\section{MATERIALS AND METHODS}

The sera of inhabitants from a town in Fukuoka Prefecture, Japan, known to have a high HCV incidence $[8,9]$ were used. From 667 subjects judged as anti-HCV positive by HCV PHA 2nd Generation Neutralization $^{\mathrm{TM}}$ (Dinabot Co., Ltd., Tokyo, Japan) or Hepatitis B surface antigen (HBs Ag) positive by 
QUICK TEST ${ }^{\mathrm{TM}}$ (Mizuho Medy Co., Ltd., Tokyo, Japan) at 1991-1994 screenings for liver disease (624 anti-HCV positive, $56 \mathrm{HBs} \mathrm{Ag}$ positive, and 13 double infection), 357 subjects were re-examined for liver diseases during January-March 1996.

To evaluate HCV infection, third generation antiHCV test kits including: SYNPEP ${ }^{\mathrm{TM}}$ HCV-EIA II (Kyokuto Pharmaceutical Inc., Tokyo, Japan) and ORTHO HCV III ELISA Test System ${ }^{\text {TM }}$ (OrthoClinical Diagnostic K.K., Tokyo, Japan) were used. A second generation anti-HCV test kit facilitating passive hemagglutination, HCV PHA 2nd Generation Neutralization $^{\mathrm{TM}}$ (Dinabot Co., Ltd., Tokyo, Japan) was also used. To detect HCV RNA, AMPLICOR ${ }^{\mathrm{TM}}$ (Nippon Roche K.K., Tokyo, Japan) was used. To evaluate different results of anti-HCV status with different kits, conformation tests with SYNPEP ${ }^{\mathrm{TM}}$ HCV-EIA II or CHIRON RIBA HCV Test System 2nd Generation ${ }^{\mathrm{TM}}$ (Ortho-Clinical Diagnostics K.K.) were performed.

SYNPEP II is a third generation antibody assay kit that detects HCV-related antibodies using enzyme immunoassay (EIA) based on a sandwich technique. The kit has 3 reaction steps: 1) a reaction between $\mathrm{HCV}$ antigens fixed in a micro-well (synthetic peptides derived from core, NS3, NS4/core, and NS5) and HCV-related antibodies in the test samples; 2) a labeling reaction with enzyme labeled anti-human $\mathrm{IgG}$; 3) a colorimetric reaction by enzyme and the substrate. The reaction time for each step is 30,15 and $15 \mathrm{~min}$, respectively; total reaction time is one hour. Antibody titers were expressed as sample/cut off value (cut off index; COI).

\section{RESULTS}

Clinical diagnosis, anti-HCV and HCV RNA detection

Table 1 shows diagnosis, positive ratio of antiHCV detected by each test kit and HCV RNA detection rate. Clinical diagnosis was based on past history, careful interview of alcohol consumption, liver function tests, abdominal ultrasound examination, physical findings, PHA results at the initial screening and HCV RNA assay using AMPLICOR ${ }^{\mathrm{TM}}$. Antibody positive ratios in 14 patients with HCVrelated liver cirrhosis and 213 with chronic hepatitis $\mathrm{C}$ were $100 \%(14 / 14)$ and $99.5 \%(212 / 213)$ by SYNPEP II, respectively: and $100 \%(14 / 14)$ and $100 \%(213 / 213)$ by ORTHO III and PHA. HCV RNA was detected in all 14 with HCV-related liver cirrhosis and in 185 (86.9\%) of the 213 with chronic hepatitis C. These results may have been influenced by following conditions: chronic hepatitis $\mathrm{C}$ was diagnosed lacking of liver histological findings; antiHCV positive subjects with normal aspartate aminotransferase (AST) and alanine aminotransferase (ALT), the subjects treated with interferon (IFN) and the subjects whose serum HCV RNA that disappeared spontaneously were included.

Of 10 subjects with no abnormality (anti-HCV positive in the initial examination by PHA, but anti$\mathrm{HCV}$ negative and normal liver function tests in the current examination), $4(40 \%)$ and $5(50 \%)$ were positive for anti-HCV when tested by SYNPEP II and ORTHO III, respectively.

TABLE 1.

Positive rate of anti-HCV and HCV RNA in various liver diseases

\begin{tabular}{|c|c|c|c|c|c|}
\hline \multirow{2}{*}{ Diagnosis } & \multirow{2}{*}{$\begin{array}{c}\text { NO. of } \\
\text { Cases }\end{array}$} & \multicolumn{4}{|c|}{ Positive Rate (NO. of Positive case) } \\
\hline & & SYNPEP II & ORTHO III & PHA & HCV RNA \\
\hline Liver Chirrhosis* & 14 & $100 \%(14)$ & $100 \%(14)$ & $100 \%(14)$ & $100 \%(14)$ \\
\hline Chronic Hepatitis* & 213 & $99.5 \%(212)$ & $100 \%(213)$ & $100 \%(213)$ & $86.9 \%(185)$ \\
\hline HCV Carrier & 74 & $94.6 \%(70)$ & $98.6 \%(73)$ & $69.0 \%$ ( 69) & $0 \%(0)$ \\
\hline $\mathrm{ALD}^{* *}$ & 11 & $90.9 \%(11)$ & $100 \%(11)$ & $100 \%(11)$ & $45.5 \%(5)$ \\
\hline Fatty Liver & 5 & $80.0 \%(4)$ & $100 \%(5)$ & $80.0 \%(4)$ & $0 \%(0)$ \\
\hline No Abnormality*** & 10 & $40.0 \%(4)$ & $50.0 \%(5)$ & $0 \%(0)$ & $0 \%(0)$ \\
\hline Total & 327 & $96.0 \%(314)$ & $98.2 \%(321)$ & $95.1 \%(311)$ & $62.4 \%(204)$ \\
\hline
\end{tabular}

All subjects were anti-HCV positive at first screening of liver diseases

* : Including subjects with normal ALT level

** : Alcoholic liver disease, alcohol consumption $>75$ g/day

*** : Anti-HCV negative by PHA at second screening of liver disease 
TABLE 2.

The Demograph of 21 cases with different results by each kit

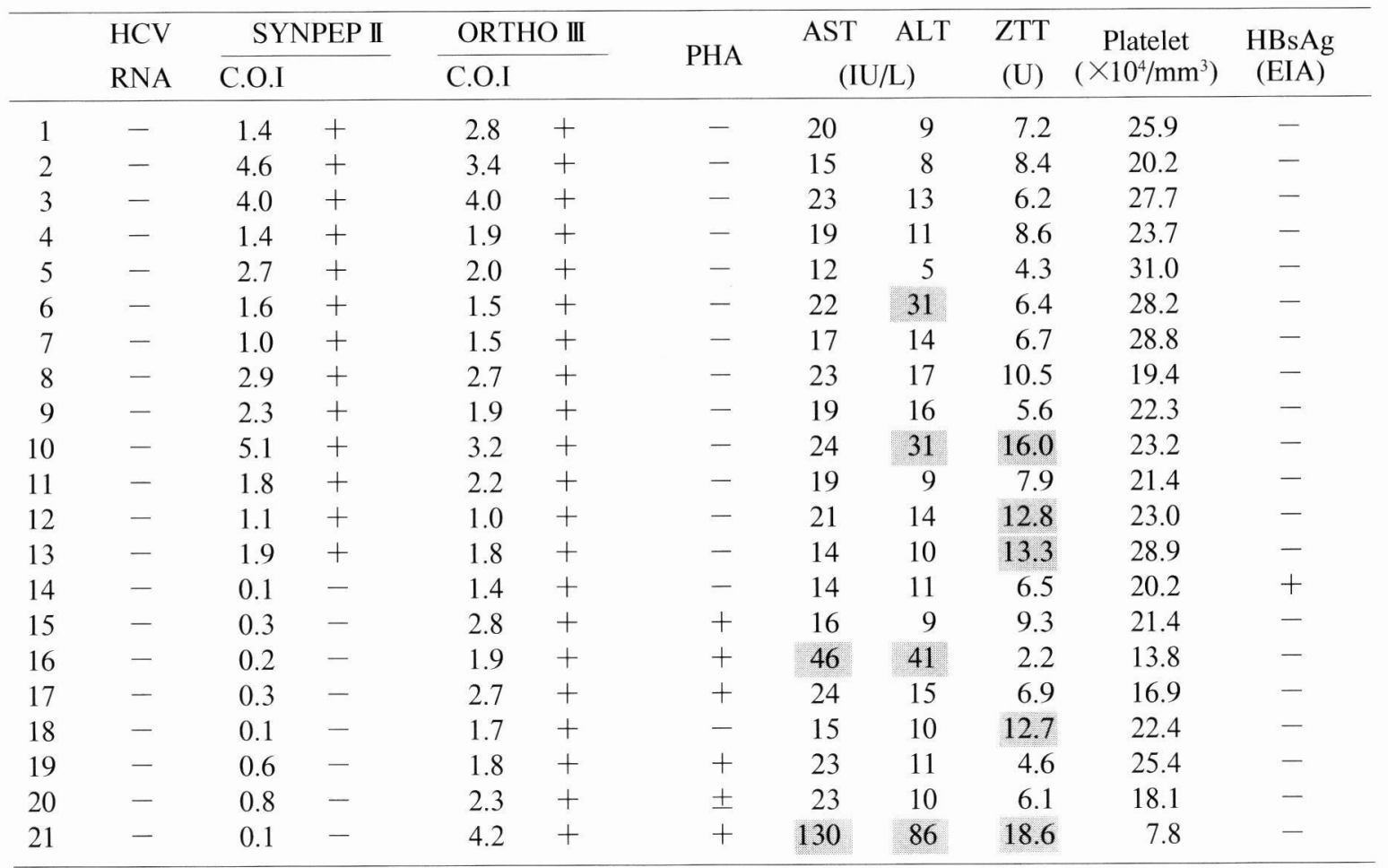

: Subjects with abnormalities in at least one of ALT, AST, or ZTT

Evaluation of discrepancy between the results of different kits

Of 339 samples, 21 had different results of antiHCV status by SYNPEP II, ORTHO III or PHA, these 21 subjects were HCV RNA negative (Table 2). Of the 21 subjects, $13(86.7 \%)$ were judged antibody positive by SYNPEP II or ORTHO III; $4(30.8 \%)$ of the 13 had slightly elevated ALT and ZTT level. One subject (No. 14), anti-HCV positive by ORTHO III, was also HBs Ag positive. Four subjects (No. 15-18), anti-HCV negative by SYNPEP II but positive by ORTHO III were assayed by RIBA-2 and by conformation kit supplied with SYNPEP II. Two anti-HCV negative subjects (No. 19 and 20) with COI of 0.6 and 0.8 by SYNPEP II resulted in borderline and negative by RIBA-2, respectively. Although these 2 subjects were anti-HCV negative by RIBA-2, they had faint bands suggesting positive for anti-HCV. For No. 21, confirmation by SYNPEP II and RIBA-2 resulted in borderline and antibody negative, respectively. This subject also had elevated AST, ALT and ZTT level. This subject needs to be followed for liver disease.

\section{Concordance among test kit results}

Table 3 shows the concordance between each kit's results. SYNPEP II had concordance with $97.6 \%$ with ORTHO III, and $90.0 \%$ with PHA, indicating

TABLE 3.

Concordance of the results between SYNPEP II ORTHO III and PHA

\begin{tabular}{|c|c|c|c|c|}
\hline \multirow{2}{*}{\multicolumn{2}{|c|}{ ORTHO III }} & \multicolumn{2}{|c|}{ Anti-HCV } & \multirow{2}{*}{ TOTAL } \\
\hline & & + & - & \\
\hline \multirow{3}{*}{ Anti-HCV } & + & 316 & 8 & 324 \\
\hline & - & 0 & 15 & 15 \\
\hline & TOTAL & 316 & 23 & 339 \\
\hline
\end{tabular}

\begin{tabular}{|c|c|c|c|c|}
\hline \multirow{2}{*}{\multicolumn{2}{|c|}{ PHA }} & \multicolumn{2}{|c|}{ Anti-HCV } & \multirow{2}{*}{ TOTAL } \\
\hline & & + & - & \\
\hline \multirow{4}{*}{ Anti-HCV } & + & 288 & 5 & 293 \\
\hline & \pm & 15 & 1 & 16 \\
\hline & - & 13 & 17 & 30 \\
\hline & TOTAL & 316 & 23 & 339 \\
\hline
\end{tabular}




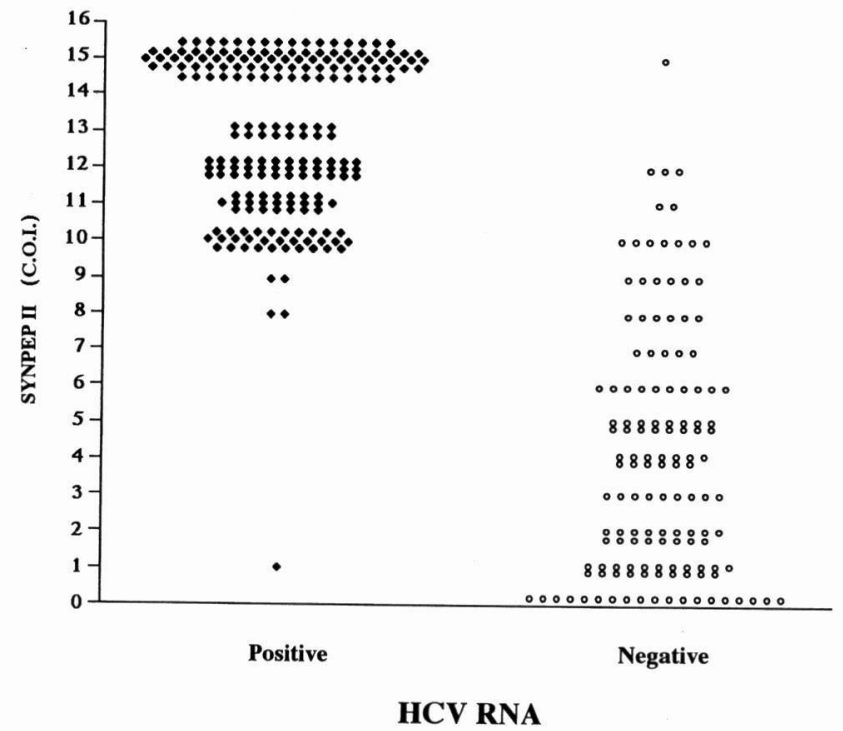

Fig. 1. Correlation between HCV RNA status (positive or negative) and cut off index (COI) of SYNPEP II.

better concordance ratio with the third generation test kit.

\section{Relationship between anti-HCV and HCV RNA status}

HCV RNA was detected in 204 subjects. AntiHCV was also positive in 204 by SYNPEP II, ORTHO III or PHA. One of the 204 had a COI of 1.0 by SYNPEP II, borderline anti-HCV positive, and also was weakly positive by ORTHO III. This subject had normal ALT level (16 IU/1). Figure 1 shows the correlation between HCV RNA detection and COI by SYNPEP II. Except one sample with COI of 1.0, COI was higher (greater than 7.5) in HCV RNA positive subjects than those in HCV RNA negative. HCV RNA negative subjects with high COI by SYNPEP II also had high COI by other kits. Those with COI of 7.5 or more by SYNPEP II had an HCV RNA positive ratio of $89.0 \%(203 / 228)$, while those with COI of less than 7.5 had an HCV RNA negative ratio of $99.1 \%(110 / 111)$.

\section{DISCUSSION}

SYNPEP II, a third generation test kit, employing a synthetic peptide as the antigen, was reportedly to have less non-specific reactions comparing other kits using recombinant antigens [10]. Also SYNPEP II needs one hour for total reaction.

SYNPEP II had a slightly lower anti-HCV detec- tion rate than ORTHO III, however, was showed to have a higher reproducibility than ORTHO III through the results of confirming test. SYNPEP II tended to have higher anti-HCV detection rates in HCV RNA negative subjects than by PHA, which is frequently used for anti-HCV screening. SYNPEP II also correlated with HCV RNA detection, therefore, it was considered to be an efficient third generation anti-HCV assay system.

The anti-HCV detection system has progressed from second to third generation with improvement in sensitivity and specificity. But, it should be recognized that even third generation anti-HCV test kit do not always detect HCV infection. Furthermore, third generation kits show discrepancies among test kits and these discrepancies may be due to the different antigen regions used. When applying these kits to clinical field, it is necessary to understand the characteristics of each kit.

From epidemiological survey, $20-30 \%$ of anti$\mathrm{HCV}$ positive persons were negative for serum $\mathrm{HCV}$ RNA. In the chronic hepatitis C patients with complete response to IFN therapy, the serum HCV RNA disappeared and antibody titers gradually decrease over the clinical course. It is useful if antibody titers are correlated with existence with serum HCV RNA. We found that approximately $90 \%$ of subjects with COI of 7.5 or greater by SYNPEP II had HCV RNA. Determining $\mathrm{HCV}$ RNA presence to confirm HCV infection is not suitable for mass screening due to its complicated procedure and high cost. SYNPEP II with cheap cost and simple procedure could be applied to mass screening to find out HCV RNA positive persons who may need clinical assistance.

\section{REFERENCES}

1. Kiyosawa K, Sodeyama T, Tanaka E, Gibo Y, Yoshizawa $\mathrm{K}$ et al. Interrelationship of blood transfusion, non-A, non-B hepatitis and hepatocellular carcinoma: analysis by detection of antibody to hepatitis $\mathrm{C}$ virus. Hepatology 1990; 12:671-675.

2. Nishioka K, Watanabe J, Furuta S, Tanaka E, Iino S et al. A high incidence of antibody to the hepatitis $\mathrm{C}$ virus in patients with hepatocellular carcinoma in Japan. Cancer 1991; 67:429-433.

3. Kiyosawa K, and Furuta S. Review of hepatitis C in Japan. J Gastroenterol Hepatol 1991; 6:383-391

4. Choo QL, Kuo G, Weiner AJ, Overby LR, Bradley DW et al. Isolation of a DNA clone derived from a bloodborne non A non B genome. Science 1989; 244:359-362.

5. Kuo G, Choo QL, Alter HJ, Gitnick GL, Redeker AG et al. An assay for circulation antibodies to a major etiologic virus of human non A non B hepatitis. Science 
$1989 ; 244: 362-364$.

6. Watabe J. Screening of anti-HCV in blood donors and preventive effects against post-transfusion hepatitis C. Jpn J Clin Med 1995; 53:395-401.

7. Katayama T. Introduction of screening for anti-HCV and post-transfusion hepatitis. Jpn J Clin Med 1995; 53:383388.

8. Noguchi S, Sata M, Suzuki H, Mizokami M, and Tanikawa K. Routes of hepatitis C virus infection in an endemic rural area of Japan; Molecular epidemiological study of hepatitis $\mathrm{C}$ virus infection. Scan J Infect Disease 1997; 29:23-28.

9. Yamakawa Y, Sata M, Suzuki H, Noguchi S, and Tanikawa K. Higher elimination rate of hepatitis $\mathrm{C}$ virus among women. J Viral Hepatitis 1996; 3:317-321.

10. Omata M. Clinical evaluation of a third generation antiHCV assay kit, SYNPEP HCV-EIA II. Kan Tan Sui 1996; 32:419-427. (in Japanese) 